\title{
ЧИСЛО СЛУЧАЕВ ДИАБЕТА ТИПА 1 И 2, ДИАГНОСТИРОВАННЫХ В АМАРА́ В ПЕРИОД С 2007 ПО 2012 гОД
}

\section{ОРИГИНАЛЬНАЯ СТАТЬЯ}

SOUZA, Kauê de Melo', FACCO, Lucas², FECURY, Amanda Alves³, ARAÚJO, Maria Helena Mendonça de ${ }^{4}$, OLIVEIRA, Euzébio de ${ }^{5}$, DENDASCK, Carla Viana ${ }^{6}$, SOUZA, Keulle Oliveira da ${ }^{7}$, DIAS, Claudio Alberto Gellis de Mattos ${ }^{8}$

SOUZA, Kauê de Melo. Et al. Число случаев диабета типа 1 и 2, диагностированных в Атара́ в период с 2007 по 2012 год. Revista Científica Multidisciplinar Núcleo do Conhecimento. 05-й год, Эд. 12, Vol. 01, стр. 18-26. Декабрь 2020 года. ISSN: 2448-0959, Ссылка доступа: https://www.nucleodoconhecimento.com.br/здравоохранение/число-случаевдиабета, DOI: 10.32749/nucleodoconhecimento.com.br/ru/66740

\section{PEЗЮME}

Сахарный диабет является последовательность различных типов расстройств в обмене веществ, которые характеризуются вызывая высокий уровень сахара в крови. Потому что это заболевание с генетическими факторами типа 1 диабет имеет в качестве основного фрактора риска ее ею, в то время как диабет типа 2,

\footnotetext{
${ }^{1}$ Горный техник, в качестве министра в Федеральном институте Amapá (IFAP).

${ }^{2}$ Студент медицинского курса Федерального университета Amapá (UNIFAP).

${ }^{3}$ Биомедицинская, кандидат биологических наук в области тропических болезней, профессор и исследователь медицинского курса Федерального университета Amapá (UNIFAP).

${ }^{4}$ Врач, профессор и исследователь медицинского курса Федерального университета Amapá (UNIFAP).

${ }^{5}$ Биолог, кандидат медицинских наук по актуальным заболеваниям, профессор и исследователь курса физкультуры Федерального университета Pará (UFPA).

${ }^{6}$ Богослов, кандидат психологических наук, исследователь Центра исследований и перспективных исследований - СЕРА.

${ }^{7}$ Социолог, студент магистратуры по антропогенным исследованиям в Амазонии, член исследовательской группы «Лаборатория образования, окружающей среды и здоровья» (LEMAS/UFPA). ${ }^{8}$ Биолог, кандидат теоретических и летных исследований, профессор и исследователь Высшей программы профессионального и технологического образования (PROFEPT), Федеральный институт Amapá (IFAP).
}

RC: 66740

Доступно в: 
кроме этих фракторов, включает ожирение, высокое кровяное давление, плохое образование питания и повышение возраста. Это исследование призвано показать количество случаев диабета типа 1 и 2, диагностированных в Амапа с переменным полом, возрастной группой, малоподвижным образом жизни, избыточным весом, курением, в период с 2007 по 2012 год. Данные исследования были взяты из компьютерного отдела SUS, DATASUS (http://datasus.saude.gov.br). Сахарный диабет 1 типа и 2 (DM1 и DM2) являются заболеваниями, которые связаны с нарушениями в производстве или в эфффективном использовании инсулина. Курение, а также малоподвижный образ жизни и избыточный вес являются важными факторами риска для развития DM2. Сахарный диабет 2 типа обеспечивает развитие различных органических поражений нерва. Кроме того, DM2, через свою хронику, позволяет развитие ретинопатии, нефропатии и других условий, негативных для здоровья человека.

Ключевые слова: Аmaра́, Сахарный диабет, избыточный вес, малоподвижный образ жизни, курение.

\section{ВВЕДЕНИЕ}

Сахарный диабет является чередой различных типов нарушений обмена веществ, которые характеризуются вызывая высокий уровень сахара в крови, как правило, связанные с отсутствием инсулина или дефицит в поглощении же тела (ADA, 2014).

Диабет типа 1 (DM1) возникает, когда организм атакует клетки, ответственные за выработку инсулина, что приводит к полному дефициту этого гормона в организме. Это происходит реже, потому что это обычно связано с генетическими фракторами. Диабет 2 типа (DM2) происходит, когда организм не может поглощать инсулин, вырабатываемый в организме из-за неспособности поджелудочной железы производить его в достаточном количестве. Это обычно происходит, когда человек имеет историю плохого питания и сидячего образа

$\mathrm{RC}: 66740$

Доступно в: 
жизни и легче приобрести, если он / она имеет наследственную склонность к болезни (BRASIL, 2006; MORA et al., 2015).

Симптомы диабета типа 1 и 2 включают увеличение объема мочи, избыточный головной загон, увеличение голода, потеря веса, усталость, перепады настроения, гипогликемия и гипергликемия. Диабет 2 типа не может представлять симптомов в течение нескольких лет, с увеличением дефицита инсулина является предохранителем для эволюции этого типа (UFRGS, 2016; NOGUEIRA et al., 2015).

Потому что это заболевание с генетическими фракторами диабета типа 1 имеет в качестве основного фрактора риска отечность, в то время как диабет типа 2, кроме этих фракторов, включает ожирение, высокое кровяное давление, плохое образование пищи и повышение возраста (LIMA et al.; 2014)

Профрилактика диабета 2 типа напрямую связана с жизнью с хорошими привычками в еде и регулярными фризическими упражнениями. Потому что это наследственное и аутоиммунное, это не так, как предотвратить диабет типа 1 (MAGALHÃES et al., 2017).

Поскольку DM1 характеризуется полным дефицитом инсулина, инсулинотерапия показана как лучшее лечение. DM2, потому что это неспособность организма производить или поглощать достаточно инсулина, лечение посвящено поддержанию гликемического контроля над телом, который включает в себя как устные лекарства и физические упражнения вместе со сбалансированной диетой (UFRGS, 2016; SANTOS; FREITAS; PINTO, 2014).

В 2012 году число случаев диабета в мире составило около 200 миллионов, в то время как в Бразилии было около 10 миллионов (BRASIL, 2012).

$\mathrm{RC}: 66740$

Доступно в: 


\section{ЦЕЛЬ}

Показать число случаев диабета типа 1 и 2, диагностированных в Амапа с переменным полом, возрастной группой, малоподвижным образом жизни, избыточным весом, курением, в период с 2007 по 2012 год.

\section{МЕТОД}

Данные взяты из ИТ-отдела SUS, DATASUS (http://datasus.saude.gov.br), следуя инструкциям: сначала была выбрана вкладка «Доступ к информации», а затем вариант «информация о здоровье (ТАБЛИЦА)» сразу после подварианта «Эпидемиология и заболеваемость» была открыта группа опций «Гипертония и диабет (ГИПЕРДИА)». Затем был выбран значок «Гипердиа - Регистрация и мониторинг пациентов с гипертонической болезнью и диабетом - с 2002 года», открыта вкладка «Выбрать вариант или щелкнуть по карте» и выбрана опция «Amapá». - если в строке поле - вариант «пол», в поле столбца - вариант «не активен», а в поле содержимого - вариант «диабет 2 типа», затем в «доступные периоды» были собраны данные с 2007 по 2012 год; это тот же период, что и для всех других коллекций. В поле строки был выбран вариант «возрастная группа», в поле столбца - вариант «не активен», а в поле содержимого вариант «диабет 2 типа». В поле строки был выбран вариант «пол», в поле столбца - вариант «не активен», а в поле содержимого - вариант «диабет 1 типа». Вариант возрастной группы был выбран в поле строки, в поле столбца вариант «не активен», а в поле содержимого - вариант «диабет 1 типа». В поле строки был выбран вариант «год», в поле столбца - вариант «не активен», а в поле содержимого - вариант «диабет 1 типа». В поле строки был выбран вариант «год», в поле столбца - вариант «не активен», а в поле содержимого вариант «диабет 2 типа». В поле строки был выбран вариант «курение», в поле столбца - вариант «не активен», а в поле содержимого - вариант «диабет 1 типа». В поле строки был выбран вариант «курение», в поле столбца - вариант «не активен», а в поле содержимого - вариант «диабет 2 типа. Сбор данных

$\mathrm{RC}: 66740$

Доступно в: 
производился в приложении Excel, компоненте Microsoft Office. пакет Corporation. Библиографическое исследование научных статей проводилось с использованием компьютеров компьютерной лаборатории Федерального института образования, науки и технологий Amapá, Campus Macapá, расположенной по адресу: Rodovia BR 210 KM 3, s / n - Bairro Brasil Novo, CEP : 68.909-398, Macapá, Amapá, Brazil.

\section{РЕЗУЛЬТАТЫ}

На рисунке 1 показано число подтвержденных случаев диабета в штате Amapá в период с 2007 по 2012 год. Было диагностировано больше случаев диабета 2 типа, чем 1 типа.

На рисунке 1 показано число подтвержденных случаев диабета в состоянии Amapá в период с 2007 по 2012 год.

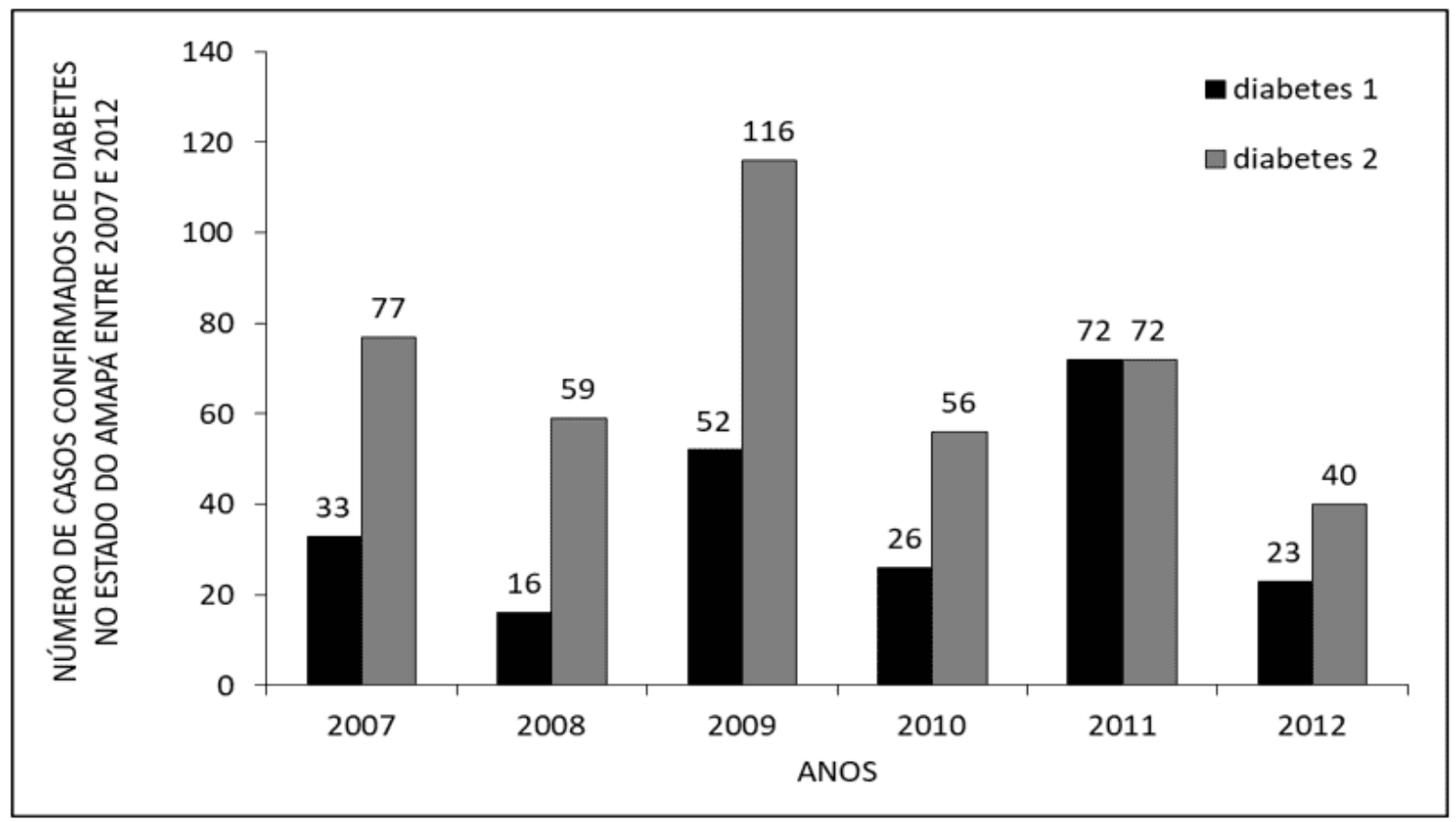

RC: 66740

Доступно в: 
На рисунке 2 показано число подтвержденных случаев диабета типа 1 и 2 в штате Amaра́ в период с 2007 по 2012 год в зависимости от пола. Наибольшее число диагнозов диабета типа 1 и 2 было у женщин.

На рисунке 2 показано число подтвержденных случаев диабета типа 1 и 2 в штате Amaра́ в период с 2007 по 2012 год в зависимости от пола.

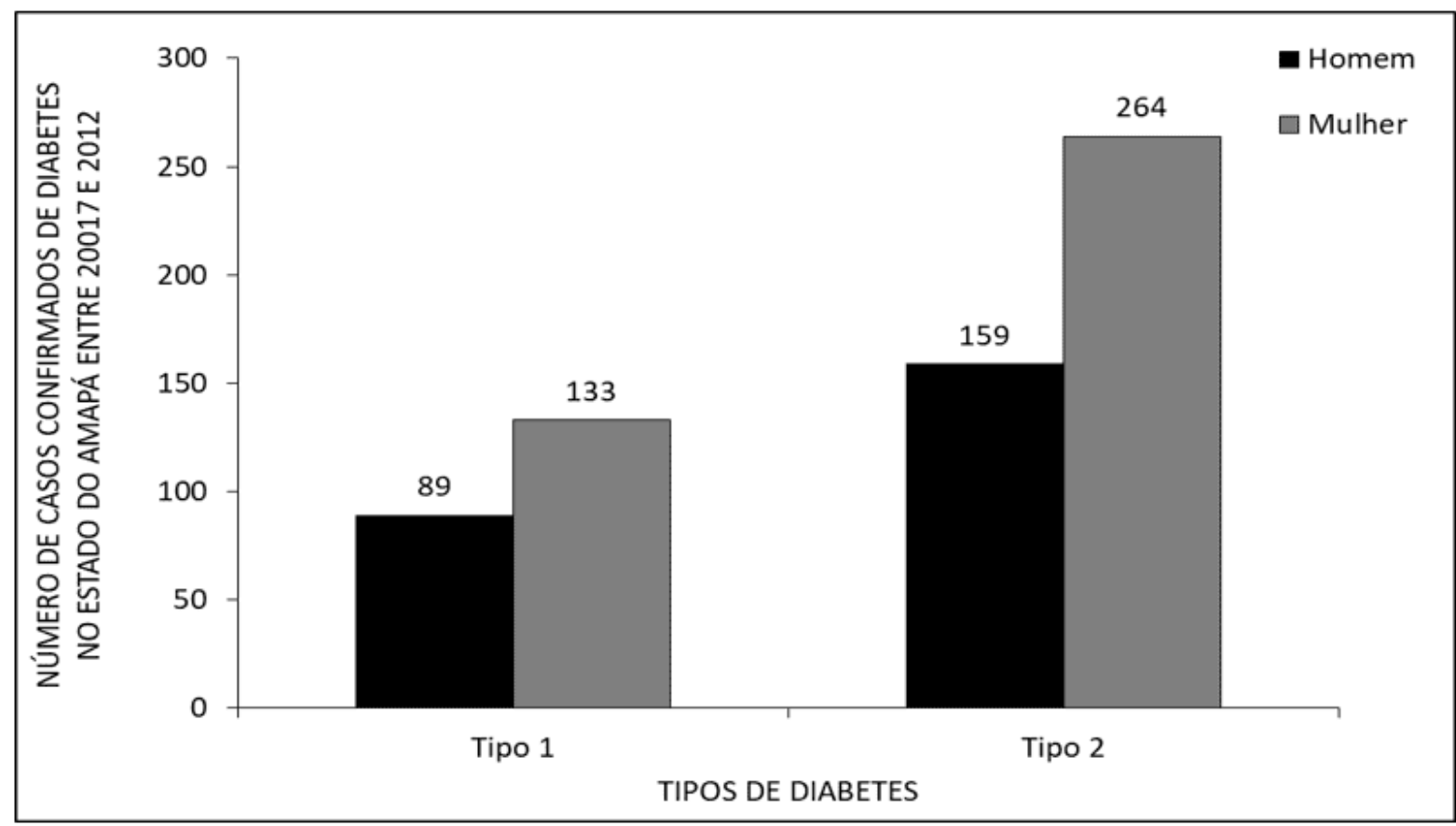

На рисунке 3 показано число подтвержденных случаев диабета типа 1 и 2 в штате Amaра́ в период с 2007 по 2012 год в зависимости от возрастной группы. Наибольшее число диагнозов диабета типа 1 и 2 было у людей в возрасте 3059 лет, в то время как самым низким было число людей в возрасте до 29 лет.

RC: 66740

Доступно в: 
На рисунке 3 показано число подтвержденных случаев диабета типа 1 и 2 в штате Amaра́ в период с 2007 по 2012 год в зависимости от возрастной группы.

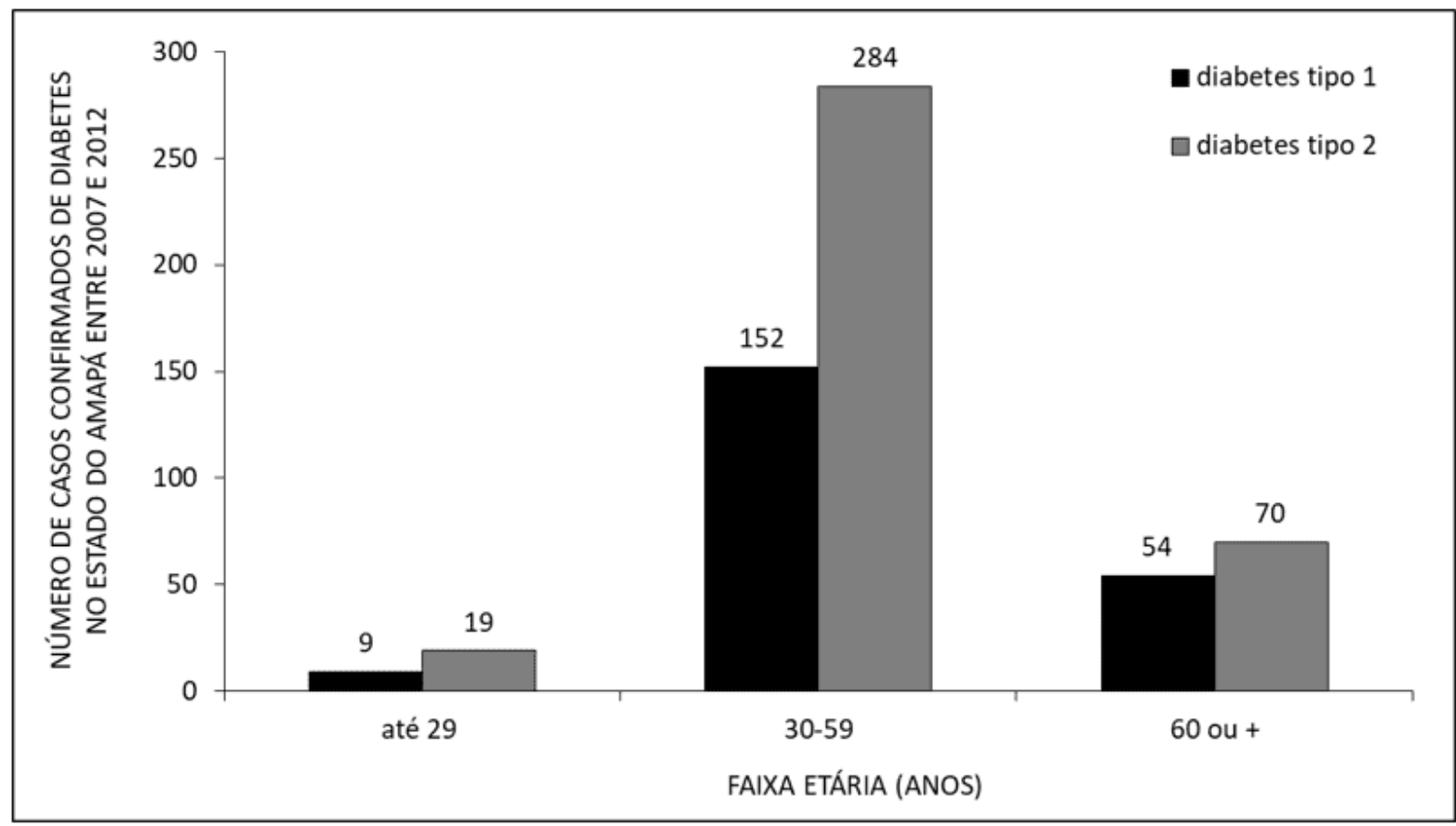

На рисунке 4 показано число подтвержденных случаев диабета типа 1 и 2 в штате Атара́ в период с 2007 по 2012 год в зависимости от веса. Наибольшее число диагностированных случаев диабета типа 1 и 2 было у людей с избыточным весом.

RC: 66740

Доступно в: 
На рисунке 4 показано число подтвержденных случаев диабета типа 1 и 2 в штате Amapá в период с 2007 по 2012 год в зависимости от веса.

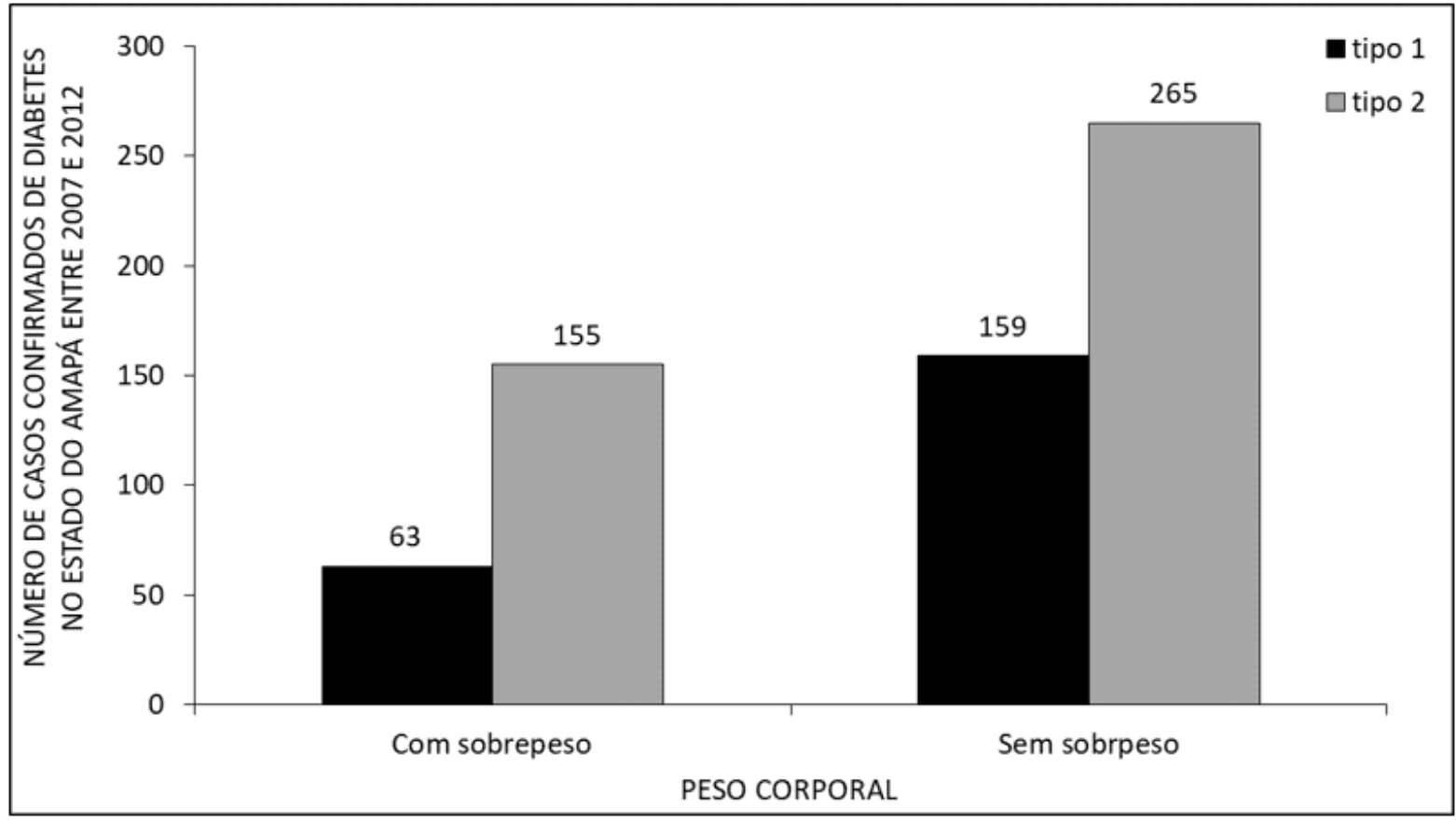

На рисунке 5 показано число подтвержденных случаев диабета типа 1 и 2 в штате Amapá в период с 2007 по 2012 год в соответствии с малоподвижным образом жизни. Наибольшее число диагнозов диабета типа 1 и 2 было у неподвижных людей.

$\mathrm{RC}: 66740$

Доступно в: 
На рисунке 5 показано число подтвержденных случаев диабета типа 1 и 2 в штате Аmaра́ в период с 2007 по 2012 год в соответствии с малоподвижным образом жизни.

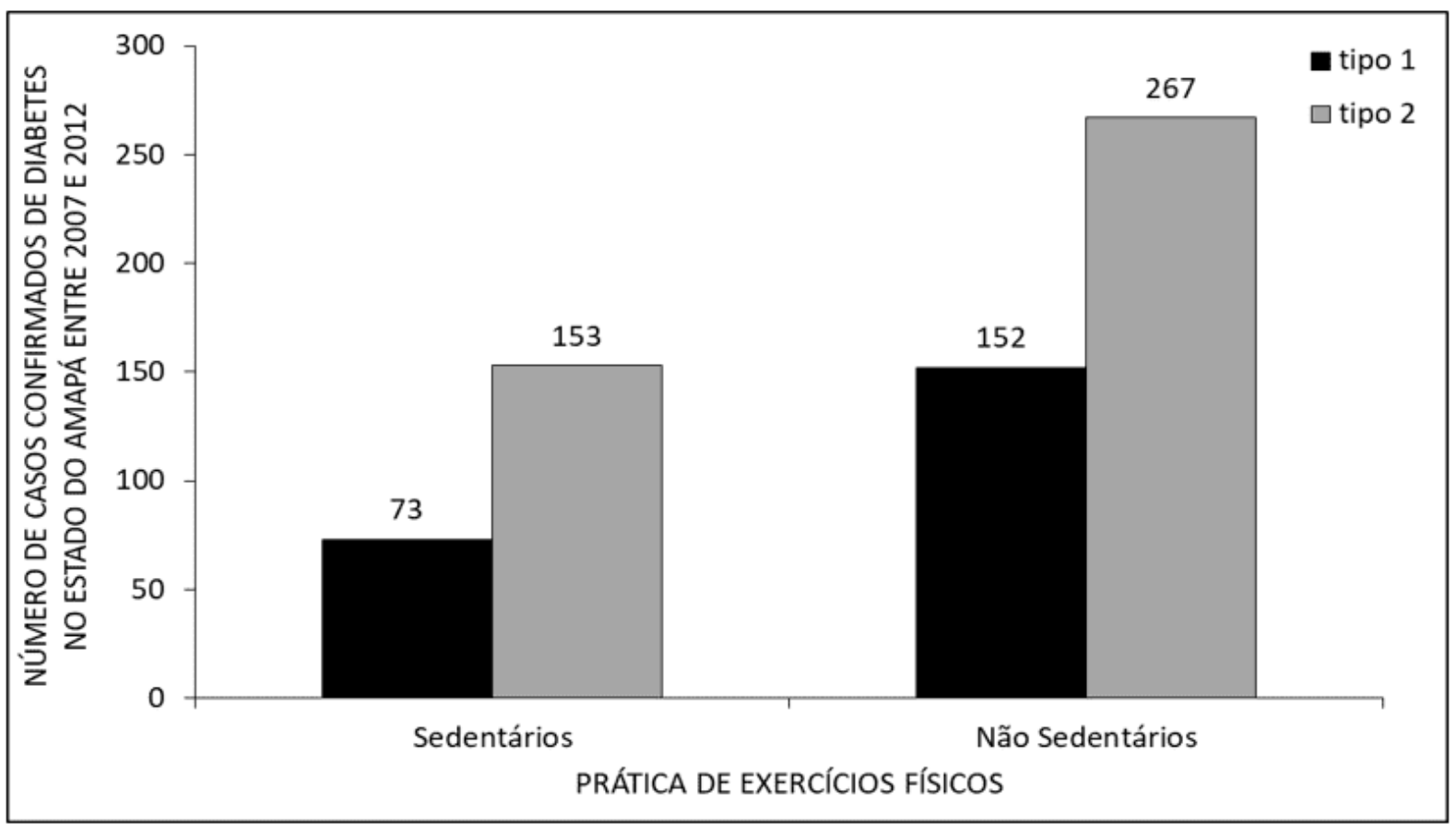

На рисунке 6 показано число подтвержденных случаев диабета типа 1 и 2 в штате Атара́ в период с 2007 по 2012 год по данным курения. Наибольшее число диагнозов диабета типа 1 и 2 было у некурящих.

RC: 66740

Доступно в: 
На рисунке 6 показано число подтвержденных случаев диабета типа 1 и 2 в штате Amapá в период с 2007 по 2012 год по данным курения.

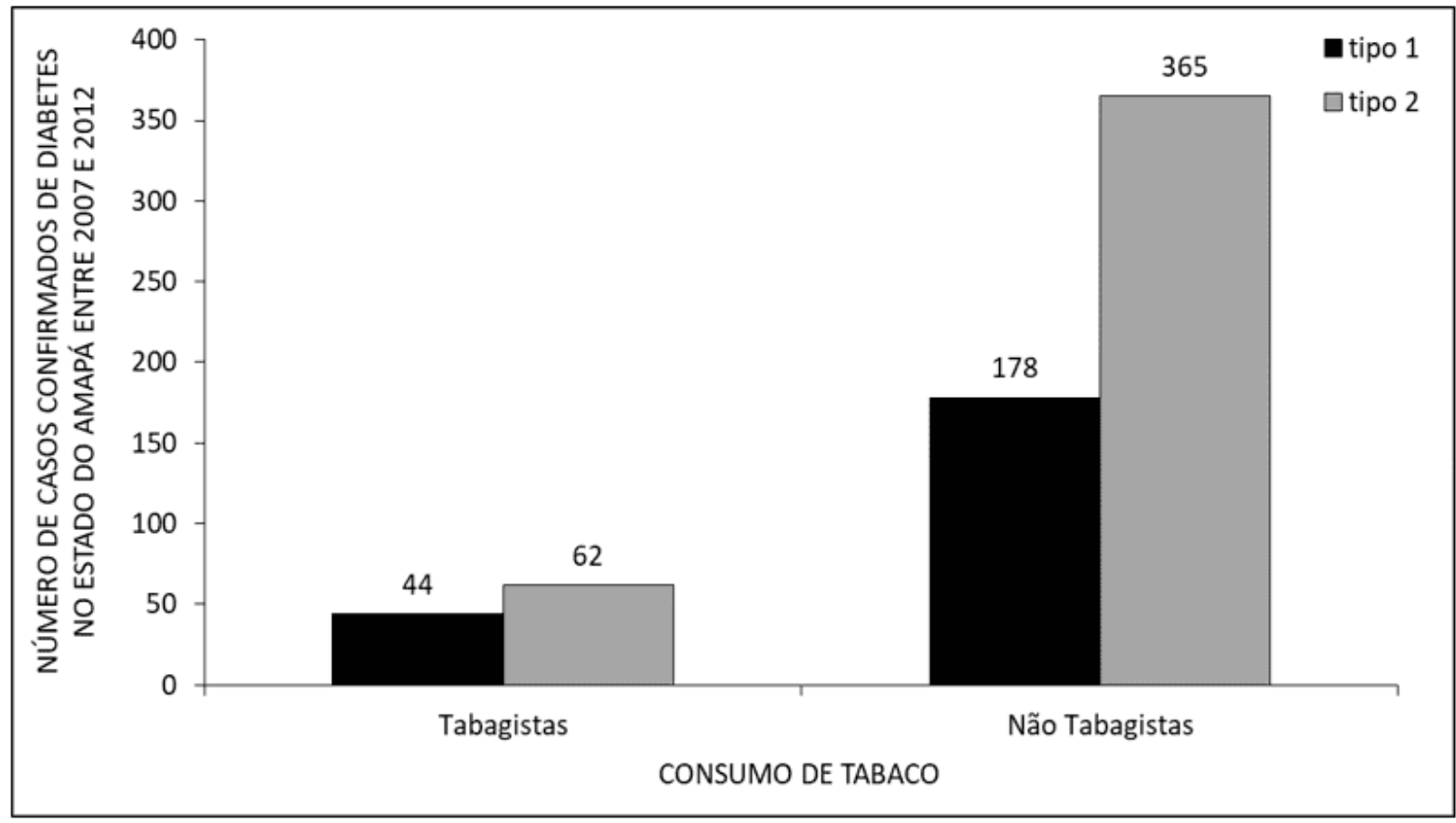

\section{ОБСУЖДЕНИЕ}

Сахарный диабет 2 типа (DM2) является одним из основных хронических неинфекционных заболеваний (НИЗ) и составляет около 90\% всех текущих случаев диабета (COSTA et al., 2017). Этот фрактор очень связан с распространенностью ожирения, так как это один из основных фракторов риска развития DM2. Число пациентов с ожирением (которые имеют DM2), которые получают конкретный и правильно структурированный уход, чтобы они могли контролировать свой вес очень низок, и, следовательно, отмечается, что эта проблема представляет собой важный фактор риска для здоровья человека, потому что ожирение представляет собой риск для развития различных заболеваний, таких как сердечно-сосудистые заболевания, влияющие в значительной степени негативным образом контроля DM2 (LIMA et al., 2015).

$\mathrm{RC}: 66740$

Доступно в: 
Отмечается, что распространенность диабета среди женщин высока, и есть несколько фракторов, которые должны быть проанализированы для этого поиска. В исследовании, проведенном в муниципалитете São Leopoldo, в Rio Grande do Sul, были оценены многочисленные параметры и переменные для этой проблемы. Было отмечено, что самая высокая распространенность диабета наблюдается среди женщин, которые имеют: возраст от 40 до 49 лет, семейное положение в браке, доход ниже 1 минимальной заработной платы, от 1 до 3 детей, практика курения, системная артериальная гипертензия (САГ), и другие фракторы, такие как ожирение, которое является наиболее важным фрактором риска для DM2 (DIAS-DA-COSTA et al., 2020).

Сахарный диабет 1 типа (DM1), также называемый инсулинозависимым диабетом, встречается чаще у пациентов-подростков, и его патофизиология включает в себя разрушение бета-клеток поджелудочной железы , ответственных за выработку инсулина в организме - и, следовательно, приводит организм к дефициту производства инсулина, что делает человека зависимым от использования синтетического инсулина. DM2 обычно происходит после 30 лет, будучи более распространенным у людей в возрасте от 50 до 60 лет. (ABREU, 2017). Его патофизиология связана с резистентностью к инсулину и, следовательно, гипогликемическое действие, выполняемое этим гормоном, не происходит адекватно, вызывая увеличение производства глюкозы печенью, способствуя широкому количеству инсулина на уровне крови (BERTONHI и DIAS, 2018).

Хотя в период с 2007 по 2012 год число подтвержденных случаев DM1 и DM2 в Amapá было выше среди людей с избыточным весом, неподвижным и некурящим, связь между DM2 и этими условиями хорошо известна. Значительная часть пациентов с сахарным диабетом 2 типа страдают ожирением или избыточным весом. Курение практика несет ответственность за экспоненциальное увеличение возможности отдельных развивающихся неоплазмы, будучи (в изоляции) основной причиной рака во всем мире. Диабет

$\mathrm{RC}: 66740$

Доступно в: 
имеет интимную связь с раком, так как он увеличивает возможность развития неоплазмы печени, в дополнение к раку толстой кишки, эндометрия, молочной железы и поджелудочной железы. Таким образом, отмечается предрасположенность курильщиков-диабетиков к неоплазме. Кроме того, сидячий образ жизни характеризуется как одна из переменных, которые следует рассматривать для развития DM2. В исследовании, проведенном со студентами из Федерального университета Ceará (UFC), было отмечено, яростное отсутствие регулярных фризических упражнений, иногда оправдано изза отсутствия мотивации и времени, в дополнение к усталости от ежедневного путешествия студента. Отсутствие ежедневной практики фризических упражнений может способствовать избыточному весу (избыточный вес и ожирение), которые являются фракторами риска для развития DM2 (LIMA et al., 2015; HOCAYEN и MALFATTI, 2010; LIMA et al., 2014).

\section{ЗАКЛЮЧЕНИЕ}

Сахарный диабет 1 типа и 2 (DM1 и DM2) являются заболеваниями, которые связаны с нарушениями в производстве или в эффективном использовании инсулина. DM1 является заболеванием, патофизиология которого не до конца известна, что включает в себя генетическую предрасположенность, в сочетании с фракторами окружающей среды. DM2, с другой стороны, даже если он имеет генетические основы, очень близок к образу жизни человека, и ожирение, избыточный вес и сидячий образ жизни являются одним из основных факторов риска для развития этой патологии.

Отмечается, что среди диабетиков распространенность женщин очень высока. Такие фракторы, как возраст от 40 до 49 лет, доход ниже 1 минимальной заработной платы, семейное положение в браке, от 1 до 3 детей, практика курения, системная артериальная гипертензия (САГ), и другие фракторы, такие как ожирение, несут ответственность за эту проблему.

$\mathrm{RC}: 66740$

Доступно в: 
DM1 встречается чаще у пациентов-подростков, и его патофизиология включает в себя разрушение бета-клеток поджелудочной железы, вызывая в организме дефицит производства инсулина, что делает человека зависимым от использования синтетического инсулина. DM2 встречается чаще после 30 лет, чаще встречается у людей в возрасте от 50 до 60 лет, и его патофизиология связана с резистентностью к инсулину и, в связи с этим, гипогликемическое действие, выполняемое инсулином, не происходит адекватно в организме, вызывая, как хроника заболевания происходит, ряд негативных последствий для организмов , с макро- и микрососудистыми поражениями и среди других.

Курение, а также малоподвижный образ жизни и избыточный вес являются важными фракторами риска для развития DM2. Сахарный диабет 2 типа обеспечивает развитие различных органических поражений нерва. Кроме того, DM2, через свою хронику, позволяет развитие ретинопатии, нефропатии и других условий, негативных для здоровья человека.

\section{ссылки}

ABREU, L. C. S. Diabetes na Terceira Idade. Revista Científica Multidisciplinar Núcleo do Conhecimento, v. 2, p. 111-131, 2017.

ADA. American Diabetes Association; Diagnosis and Classification of Diabetes Mellitus; Diabetes Care, Volume 37, Supplement 1, January 2014.

BERTONHI, L. G.; DIAS, J. C. R. Diabetes mellitus tipo 2: aspectos clínicos, tratamento e conduta dietoterápica. Revista Ciências Nutricionais Online, v. 2, n. 2, p. 1-10, 2018.

BRASIL. Ministério da Saúde. Secretaria de Atenção à Saúde. Departamento de Atenção Básica. Diabetes Mellitus / Ministério da Saúde, Secretaria de Atenção à Saúde, Departamento de Atenção Básica. - Brasília : Ministério da Saúde, 2006.

$\mathrm{RC}: 66740$

Доступно в: 
BRASIL. Portal Brasil. Diabetes traz consequências graves se não for controlado. 2012. disponível em: <http://www.brasil.gov.br/saude/2012/04/diabetes>. Acessado Em: 19/09/2017.

CORTEZ. D.N; Reis, I.A; Souza, D.A.S; Macedo, M.M.L; Torres H.C Complicações e o tempo de diagnóstico do diabetes mellitus na atenção primária Acta Paulista de Enfermagem, vol. 28, núm. 3, 2015, pp. 250-255.

COSTA, A. F.; FLOR, L. S.; CAMPOS, M. R.; OLIVEIRA, A. F.; COSTA, M. F. S.; SILVA, R. S.; LOBATO, L. C. P.; SCHRAMM, J. M. A. Carga do diabetes mellitus tipo 2 no Brasil. Cad. Saúde Pública, v. 33, n. 2, p. 1-14, 2017.

DIAS-DA-COSTA, J. S.; SILOCCHI, C.; SCHWENDLER, S. C.; MORIMOTO, T.; MOTTIN, V. H. M.; PANIZ, V. M. V.; BAIRROS, F. S.; OLINTO, M. T. A. Prevalência de diabetes mellitus autorreferido em mulheres e fatores associados: estudo de base populacional em São Leopoldo, Rio Grande do Sul, 2015. Epidemiol. Serv. Saude, v. 29 , n. 2, p. 1-12, 2020.

HOCAYEN, P. A. S.; MALFATTI, C. R. M. Tabagismo em pacientes diabéticos: predisposição às doenças crônico-degenerativas e neoplasia. Cinergis, v. 11, n. 2, p. 19-25, 2010.

LIMA, A. C. S.; ARAÚJO, M. F. M.; FREITAS, R. W. J. F.; ZANETTI, M. L.; ALMEIDA, P. C.; DAMASCENO, M. M. C. Fatores de risco para diabetes mellitus tipo 2 em universitários: associação com variáveis sociodemográficas. Rev. LatinoAm. Enfermagem, v. 22, n. 3, p. 484-490, 2014.

LIMA, L. L.; SÁ, A. D.; FIGUEIREDO, A. S.; MUÑOZ, R. L. S. Prevalência de sobrepeso e obesidade em diabéticos tipo 2 atendidos no ambulatório de Endocrinologia de um Hospital Universitário. Rev Soc Bras Clin Med, v. 13, n. 4, p. 251-256, 2015.

RC: 66740

Доступно в: 
MORA, G.P.C.; Abascal I.C.; Sanabria, G. Sobrepeso, obesidad y diabetes mellitus 2 en adolescentes de América Latina en 2000-2010;Revista Cubana de Medicina General Integral. v. 31, n. 3, 217-231, 2015.

SANTOS, M. S, Freitas, N. M; Pinto, F. O; O DIABETES MELLITUS TIPO 1 E TIPO 2 E SUA EVOLUÇÃO NO MUNICÍPIO DE QUISSAMÃ-RJ; Revista Científica Interdisciplinar, vol 1,No 1, 2014.

UFRGS. RegulaSUS. Diabetes Mellitus. Disponível em: $<$ https://www.ufrgs.br/telessauders/documentos/protocolos_resumos/endocrino_resu mo_diabetes_TSRS_20160324.pdf >.Acesso em: 19/09/2017.

Представлено: Декабрь 2020 года.

Утверждено: Декабрь 2020 года.

RC: 66740

Доступно в: 